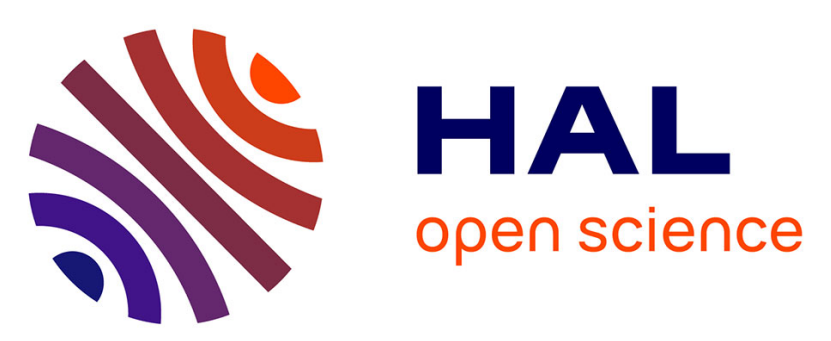

\title{
Adaptive nonlinear output feedback for transient stabilization and voltage regulation of power generators with unknown parameters
}

G. Damm, Riccardo Marino, Françoise Lamnabhi-Lagarrigue

\section{To cite this version:}

G. Damm, Riccardo Marino, Françoise Lamnabhi-Lagarrigue. Adaptive nonlinear output feedback for transient stabilization and voltage regulation of power generators with unknown parameters. International Journal of Robust and Nonlinear Control, 2004, 14 (910), pp.833-855. 10.1002/rnc.908 . hal-02936796

\section{HAL Id: hal-02936796 \\ https://hal.science/hal-02936796}

Submitted on 11 Sep 2020

HAL is a multi-disciplinary open access archive for the deposit and dissemination of scientific research documents, whether they are published or not. The documents may come from teaching and research institutions in France or abroad, or from public or private research centers.
L'archive ouverte pluridisciplinaire $\mathbf{H A L}$, est destinée au dépôt et à la diffusion de documents scientifiques de niveau recherche, publiés ou non, émanant des établissements d'enseignement et de recherche français ou étrangers, des laboratoires publics ou privés. 


\title{
Adaptive nonlinear output feedback for transient stabilization and voltage regulation of power generators with unknown parameters
}

\author{
Gilney Damm ${ }^{1, *, \dagger}$, Riccardo Marino ${ }^{2, \ddagger}$ and Françoise Lamnabhi-Lagarrigue ${ }^{3, \S}$ \\ 1 Laboratoire Systèmes Complexes, LSC-CNRS, 40 rue du Pelevoux, 91020, Evry Cedex, France \\ 2 Dip. di Ingegneria Elettronica, Università di Roma Tor Vergata, via di Tor Vergata 110, 00133 Rome, Italy \\ ${ }^{3}$ Laboratoire des Signaux et Systèmes, L2S-CNRS, Plateau de Moulon, 91192, Gif sur Yvette, France
}

\section{SUMMARY}

This work presents a nonlinear adaptive output feedback excitation control, designed for a synchronous generator modeled by a standard third order model on the basis of the physically available measurements of relative angular speed, active and reactive electric power and terminal voltage. The power angle, which is a crucial variable for the excitation control, as well as mechanical power and the impedance of the transmission line connecting the generator to an infinity bus, are not assumed to be available for feedback. The feedback control achieves transient stabilization and voltage regulation when faults occur to the turbines or the transmission lines, such that parameters (mechanical power and line impedance) may permanently take any (unknown) value. The controller recovers by adaptation the unknown values and simultaneously generates trajectories to be followed by the states, that converge to the new equilibrium point.

KEY WORDS: non-linear control; adaptive control; power system stabilization; power generators

\section{Introduction}

Power system stabilization has been dealt with for many years by both control and power systems communities. For the latter, the goal is to have stable, reliable and robust electrical energy production and distribution. On the other hand, control system teams develop quite more complicated systems which may be difficult to implement. Our goal here is to present new control methods for power system stabilization, which are closer to physical considerations. These new control methods, mainly based on modern nonlinear techniques, may improve power systems stabilization since classical controllers found in most power plants have limitations in performance and in operation region.

On the other hand, the theoretical interest of these systems becomes evident as we remark that power generators are described by nonlinear equations with unknown time varying

\footnotetext{
${ }^{*}$ Correspondence to: Gilney Damm, LSC-CNRS, 40 rue du Pelevoux, 91020, Evry Cedex, France

${ }^{\dagger}$ Email:gilney@iup.univ-evry.fr

‡Email:marino@ing. uniroma2.it

$\S$ Email:lamnabhi@lss.supelec.fr
} 
parameters. There is no full state measurement, and they are underactuated systems. All these features make the problem quite difficult and interesting from a theoretical point of view. Its classical solution is presented in [10] and [2] using robust linear techniques that are widespread in most power plants. Modern linear robust and adaptive control techniques applied to this problem, may be seen in [7], [8] and [3]. Recently, feedback linearization ( [11], [6] and [20]) as well as nonlinear adaptive techniques ([1] and [21]) were proposed to design stabilizing controllers with the purpose of enlarging the stability region of the operating condition.

The nonlinear feedback control algorithms so far proposed in the literature make use of power angle and mechanical power measurements, which are physically not available. These algorithms have also the difficulty of determining the faulted equilibrium value which is compatible with the required terminal voltage once the fault (mechanical or electrical failure) has occurred. This is our motivation to propose a nonlinear scheme based only on actually measured outputs. First, in Section 2, following the lines of our previous works [4], [12] and [5], we make use of the standard third order model used in [21] (see [2] and [19]) to show that the terminal voltage, the relative angular speed and the active electric power (which are actually measurable and available for feedback) are state variables in the physical region of the state space. We then develop an adaptive feedback linearization of the system achieving exponential stability of the closed loop system, as presented in Section 3. To do so, for a given set of unknown parameters, we recover, by adaptation, the new equilibrium point of the system and generate, on-line, a trajectory that drives the generator toward this point. This task becomes complicated as we have a nonlinear and nonlinearly-parametrized system with unknown time-varying parameters, without full state measurement. Tracking in such systems is a difficult task, and has been recently studied for the SISO case in [14]. We conclude the paper with simulations (Section 4) that show the good behavior of the adaptive controller in the presence of transmission line and turbine faults.

\section{Dynamical Model}

The power generator is represented by the standard model presented in [2] (also used in [6], [19], [20] and [21]) that may be decomposed in a mechanical and an electrical parts. The advantage of such a model is that although being of low order, it expresses well the behavior of large systems. This fact (model reduction) is well developed in [18] where a mathematical approach leads to the same conclusions of standard physical simplifications. In practice, this may be seen as the Thevenin equivalent of a large network.

Let's first consider the simplified mechanical model expressed in per unit as

$$
\begin{aligned}
\dot{\delta} & =\omega \\
\dot{\omega} & =-\frac{D}{H} \omega+\frac{\omega_{s}}{H}\left(P_{m}-P_{e}\right)
\end{aligned}
$$

where: $\delta(\mathrm{rad})$ is the power angle of the generator relative to the angle of the infinite bus rotating at synchronous speed $\omega_{s} ; \omega(\mathrm{rad} / \mathrm{s})$ is the angular speed of the generator relative to the synchronous speed $\omega_{s}$ i.e. $\omega=\omega_{g}-\omega_{s}$ with $\omega_{g}$ being the generator angular speed; $H(s)$ is the per unit inertia constant; $D(p . u$. $)$ is the per unit damping constant; $P_{m}(p . u$.) is the per unit mechanical input power; $P_{e}(p . u$.$) is the per unit active electric power delivered by the$ 
generator to the infinite bus. Note that the expression $\omega_{s}^{2} / \omega_{g}$ is simplified as $\omega_{s}^{2} / \omega_{g} \simeq \omega_{s}$ in the right-hand side of (1). The active and reactive $(Q(p . u))$ powers are given by

$$
\begin{aligned}
P_{e} & =\frac{V_{s} E_{q}}{X_{d s}} \sin (\delta) \\
Q & =\frac{V_{s}}{X_{d s}} E_{q} \cos (\delta)-\frac{V_{s}^{2}}{X_{d s}}
\end{aligned}
$$

where: $E_{q}(p . u$.$) is the quadrature's EMF; V_{s}(p . u$.$) is the voltage at the infinite bus; X_{d s}(p . u.) \triangleq$ $X_{T}+\frac{1}{2} X_{L}+X_{d}$ is the total reactance which takes into account $X_{d}(p . u$.$) , the generator direct$ axis reactance, $X_{L}(p . u$.$) , the transmission line reactance, and X_{T}(p . u$.), the reactance of the transformer. The quadrature EMF, $E_{q}$, and the transient quadrature EMF, $E_{q}^{\prime}$, are related by

$$
E_{q}=\frac{X_{d s}}{X_{d s}^{\prime}} E_{q}^{\prime}-\frac{X_{d}-X_{d}^{\prime}}{X_{d s}^{\prime}} V_{s} \cos (\delta)
$$

while the dynamics of $E_{q}^{\prime}$ (representing the electrical part of the generator) are given by

$$
\frac{d E_{q}^{\prime}}{d t}=\frac{1}{T_{d 0}}\left(K_{c} u_{f}-E_{q}\right)
$$

in which: $X_{d s}^{\prime}(p . u.) \triangleq X_{T}+\frac{1}{2} X_{L}+X_{d}^{\prime}$ with $X_{d}^{\prime}(p . u$.) denoting the generator direct axis transient reactance; $u_{f}\left(p . u\right.$.) is the input to the (SCR) amplifier of the generator; $K_{c}$ is the gain of the excitation amplifier; $T_{d 0}(s)$ is the direct axis short circuit time constant. Substituting (2) into (1) and (4) into (5), we obtain the state space model

$$
\begin{aligned}
\dot{\delta} & =\omega \\
\dot{\omega} & =-\frac{D}{H} \omega+\frac{\omega_{s}}{H}\left(P_{m}-\frac{V_{s}}{X_{d s}^{\prime}} E_{q}^{\prime} \sin (\delta)+\frac{X_{d}-X_{d}^{\prime}}{X_{d s} X_{d s}^{\prime}} V_{s}^{2} \sin (\delta) \cos (\delta)\right) \\
\dot{E}_{q}^{\prime} & =\frac{1}{T_{d 0}}\left(K_{c} u_{f}-\frac{X_{d s}}{X_{d s}^{\prime}} E_{q}^{\prime}+\frac{X_{d}-X_{d}^{\prime}}{X_{d s}^{\prime}} V_{s} \cos (\delta)\right)
\end{aligned}
$$

in which $\left(\delta, \omega, E_{q}^{\prime}\right)$ is the state and $u_{f}$ is the control input. Since $P_{e}$ is measurable while $E_{q}^{\prime}$ is not, it is convenient to express the state space model using $\left(\delta, \omega, P_{e}\right)$ as states which are equivalent states as long as the power angle $\delta$ remains in the open set $0<\delta<\pi$.

In the following, we take into account the notation

$$
T_{d 0}^{\prime}=\frac{X_{d s}^{\prime}}{X_{d s}} T_{d 0}
$$

where $T_{d 0}^{\prime}$ is the direct axis transient short circuit time constant. Differentiating (2) with respect to time, and using (1)-(5), we obtain 


$$
\begin{aligned}
\dot{\delta} & =\omega \\
\dot{\omega} & =-\frac{D}{H} \omega-\frac{\omega_{s}}{H}\left(P_{e}-P_{m}\right) \\
\dot{P_{e}} & =-\frac{1}{T_{d 0}^{\prime}} P_{e}+\frac{1}{T_{d 0}^{\prime}}\left\{\frac{V_{s}}{X_{d s}} \sin (\delta)\left[K_{c} u_{f}+T_{d 0}^{\prime}\left(X_{d}-X_{d}^{\prime}\right) \frac{V_{s}}{X_{d s}^{\prime}} \omega \sin (\delta)\right]\right. \\
& \left.+T_{d 0}^{\prime} P_{e} \omega \cot (\delta)\right\}
\end{aligned}
$$

which is valid provided that $0<\delta<\pi$. Note that when $\delta$ is near 0 or near $\pi$ the effect of the input $u_{f}$ on the overall dynamics is greatly reduced.

The generator terminal voltage is given by

$$
V_{t} e^{j \varphi}=\frac{j X_{s} E_{q} e^{j\left(\frac{\pi}{2}+\delta\right)}+j X_{d} V_{s} e^{j \frac{\pi}{2}}}{j X_{d s}}
$$

where

$$
\begin{aligned}
& X_{s}=X_{T}+\frac{X_{L}}{2} \\
& X_{d s}=X_{d}+X_{s}
\end{aligned}
$$

so that its modulus is

$$
V_{t}=\frac{1}{X_{d s}}\left(X_{s}^{2} E_{q}^{2}+V_{s}^{2} X_{d}^{2}+2 X_{s} X_{d} E_{q} V_{s} \cos (\delta)\right)^{\frac{1}{2}}
$$

or in the new state variables

$$
V_{t}=\left(\frac{X_{s}^{2} P_{e}^{2}}{V_{s}^{2} \sin ^{2}(\delta)}+\frac{X_{d}^{2} V_{s}^{2}}{X_{d s}^{2}}+\frac{2 X_{s} X_{d}}{X_{d s}} P_{e} \cot (\delta)\right)^{\frac{1}{2}}
$$

which is the output of the system to be regulated to its reference value $V_{t r}=1(p . u$.)

We must remark in this model that mechanical power, power angle and line impedance are not available for measurement. Actually, this is the main blocking point for nonlinear control of power generators.

We avoid this problem using the relation (see [4]):

$$
X_{s}=\frac{-Q V_{s}^{2} \pm \sqrt{Q^{2} V_{s}^{4}-\left(Q^{2}+P_{e}^{2}\right) V_{s}^{2}\left(V_{s}^{2}-V_{t}^{2}\right)}}{Q^{2}+P_{e}^{2}}
$$

to express the line impedance, and the relation:

$$
\delta=\operatorname{arccot}\left(\frac{V_{s}}{X_{s} P_{e}}\left(-\frac{X_{d} V_{s}}{X_{d s}}+\sqrt{V_{t}^{2}-\frac{X_{s}^{2}}{V_{s}^{2}} P_{e}^{2}}\right)\right)
$$

to express the power angle. With respect to the mechanical power, we will present an adaptive scheme to recover its value. Note that in equation (9), we use $X_{s}$ as the impedance of the 
line up to the point of the network where the voltage is equal to $V_{s}$. Errors in the infinity bus voltage will be expressed as a different value of line impedance, leading to an equivalent result for the controller.

One must also remark that (10) is a one-to-one function from $\delta$ to $V_{t}$ (as $V_{t}$ is positive). As a consequence, $\left(V_{t}, \omega, P_{e}\right)$, which are measurable and are available for feedback action, is an equivalent state for the models (6) and (7).

\section{Nonlinear Adaptive Controller}

The operating conditions $\left(\delta_{0}, \omega_{0}, P_{e 0}\right)$ of the synchronous generator model $(7)$ are given by

$$
\begin{aligned}
\omega_{0} & =0 \\
P_{e 0} & =P_{m} \\
-P_{m}+\frac{V_{s}}{X_{d s}} K_{c} u_{f} \sin (\delta) & =0
\end{aligned}
$$

Note that while $\omega_{0}=0, P_{e 0}=P_{m}$ are not affected by $u_{f}$, from the third equation above we see that there are two operating conditions $\delta_{s}, \delta_{u}, 0<\delta_{s}<\frac{\pi}{2}, \frac{\pi}{2}<\delta_{u}<\pi$ for constant inputs $u_{f}>\left(P_{m} X_{d s}\right) /\left(K_{c} V_{s}\right) ;\left(\delta_{s}, 0, P_{m}\right)$ is an asymptotically stable equilibrium point while $\left(\delta_{u}, 0, P_{m}\right)$ is an unstable equilibrium point. The stable operating condition $\left(\delta_{s}, 0, P_{m}\right)$ and the corresponding excitation constant input

$$
K_{c} u_{f 0}=\frac{P_{m} X_{d s}}{V_{s} \sin \left(\delta_{s}\right)}
$$

are chosen so that the modulus of the generator terminal voltage

$$
V_{t}=\frac{1}{X_{d s}}\left(X_{s}^{2} K_{c}^{2} u_{f 0}^{2}+V_{s}^{2} X_{d}^{2}+2 X_{s} X_{d} K_{c} u_{f 0} V_{s} \cos \left(\delta_{s}\right)\right)^{\frac{1}{2}}
$$

is equal to the prescribed value $V_{t r}$.

The objective of the control system is to keep all states and outputs bounded and asymptotically bring outputs/states to their reference values. These objectives may be summarized as:

$$
\begin{gathered}
0<\delta<180 \\
|\omega| \leq \omega_{M}<\infty \\
\left|P_{e}\right|<\infty
\end{gathered} \quad \quad \lim _{t \rightarrow \infty}\left[\begin{array}{c}
\omega \\
P_{e} \\
V_{t}
\end{array}\right]=\left[\begin{array}{c}
0 \\
P_{m} \\
V_{t r}
\end{array}\right]
$$

where $\omega_{M}$ is a limit value for the angular velocity that is specified by the constructor.

One must remark that parameters may, and will, abruptly change in time. For instance, the parameter $P_{m}$ may abruptly change to an unknown faulted value $P_{m f}$ due to turbine failures, so that $\left(V_{t r}, 0, P_{m}\right)$ may not belong to the region of attraction of the faulted equilibrium point $\left(V_{t r}, 0, P_{m f}\right)$. The state feedback control should be designed so that typical turbine failures do not cause instabilities and consequently loss of synchronism and inability to achieve voltage regulation.

Prepared using rncauth.cls

Int. J. Robust Nonlinear Control 20; :- 
A reduction from $P_{m}$ to $\left(P_{m}\right)_{f}$ of the mechanical power generated by the turbine, changes the operating condition: the new operating condition $(\delta)_{f}$ is the solution of

$$
-\frac{\left(P_{m}\right)_{f}}{P_{m}}+\frac{\sin (\delta)_{f}}{\sin \left(\delta_{s}\right)}=0
$$

and since $\left(P_{m}\right)_{f}$ is typically unknown, the corresponding new stable operating condition $\left(\delta_{s}\right)_{f}$ is also unknown. The control system must recover this new operation point, generate a trajectory towards it, and drive the system to this trajectory.

To develop the control, the model (7) is rewritten as:

$$
\begin{aligned}
\dot{\delta} & =\omega \\
\dot{\omega} & =-\frac{D}{H} \omega-\frac{\omega_{s}}{H}\left(P_{e}-\theta\right) \\
\dot{P}_{e} & =-\frac{1}{T_{d 0}^{\prime}} P_{e}+\frac{V_{s}}{X_{d s} T_{d 0}^{\prime}} \sin (\delta) K_{c} u_{f}+\frac{\left(X_{d}-X_{d}^{\prime}\right) V_{s}^{2}}{X_{d s} X_{d s}^{\prime}} \omega \sin ^{2}(\delta)+P_{e} \omega \cot (\delta)
\end{aligned}
$$

in which $\theta(t)$ is a possibly time-varying disturbance; the parameter $\theta$ is assumed to be unknown and to belong to the known compact set $\left[\theta_{m}, \theta_{M}\right]$ where the lower and upper bounds $\theta_{m}, \theta_{M}$ are known.

Let $\delta_{r}(t)$ be a (at least) $C^{3}$ reference signal (toward the new equilibrium point) to be tracked. In order to build this trajectory $\left(\delta_{r}\right)$ toward the equilibrium value of the power angle $\left(\delta_{s}\right)$, we use equation (13) where we replace $V_{t}$ by its reference value $V_{t r} ; V_{s}$ is considered as 1 (p.u.); $X_{s}$ is the impedance of the line up to the point of the network where the voltage is equal to $V_{s}$, and is calculated by (9); $X_{d}$ is a known constant and finally $P_{e}$ is replaced by $\widehat{P}_{m}$ that is the estimation of $P_{m}$. The resulting expression is:

$$
\delta_{r}=\operatorname{arccot}\left(\left(\frac{V_{s}}{X_{s} \widehat{P}_{m}}\right)\left(-\frac{V_{s}}{X_{d s}} X_{d}+\sqrt{V_{t r}^{2}-\frac{X_{s}^{2}}{V_{s}^{2}} \widehat{P}_{m}^{2}}\right)\right)
$$

As $\operatorname{arccot}(x)$ is a one-to-one smooth function, one may compute the correct $\delta_{r}$ for each set of arguments. Remark that as $\widehat{P}_{m}$ goes to $P_{m}, \delta_{r}$ goes to $\delta_{s}$.

In order to estimate $P_{m}$ we define ( $\hat{\omega}$ is an estimation of $\omega$ ):

$$
\begin{aligned}
\widetilde{P}_{m} & =P_{m}-\widehat{P}_{m} \\
\widetilde{\omega}_{e} & =(\omega-\hat{\omega})
\end{aligned}
$$

One must not confound this new defined $\widetilde{\omega}_{e}$ with variable $\widetilde{\omega}$ that we will define later. We may then write:

$$
\begin{aligned}
\dot{\widetilde{P}}_{m} & =-\dot{\widehat{P}}_{m}=-\gamma_{1} \widetilde{\omega}_{e} \\
\dot{\hat{\omega}} & =-\frac{D}{H} \hat{\omega}-\frac{\omega_{s}}{H}\left(P_{e}-\hat{P}_{m}\right)
\end{aligned}
$$

and then, using also the second equation of (7), we conclude that:

Prepared using rncauth.cls

Int. J. Robust Nonlinear Control 20; :- 


$$
\dot{\widetilde{\omega}}_{e}=-\frac{D}{H} \widetilde{\omega}_{e}+\frac{\omega_{s}}{H} \widetilde{P}_{m}
$$

or in a more concise form

$$
\left[\begin{array}{c}
\dot{\widetilde{P}}_{m} \\
\dot{\widetilde{\omega}}_{e}
\end{array}\right]=\left[\begin{array}{cc}
0 & -\gamma_{1} \\
\frac{\omega_{s}}{H} & -\frac{D}{H}
\end{array}\right]\left[\begin{array}{c}
\widetilde{P}_{m} \\
\widetilde{\omega}_{e}
\end{array}\right]
$$

which eigenvalues are:

$$
\lambda_{i}=\frac{1}{2} \frac{-D \pm \sqrt{D^{2}-4 H \omega_{s} \gamma_{1}}}{H}
$$

We may then see that a suitable choice of $\gamma_{1}$ will give an exponentially stable estimation. Actually, any $\gamma_{1}>0$ will meet this requirement, in particular a $0<\gamma_{1} \leq \frac{D^{2}}{4 H \omega_{s}}$ that will give two negative real roots.

Next, we define:

$$
\widetilde{\delta}(t)=\delta(t)-\delta_{r}(t)
$$

where, taking the time derivative, we obtain:

$$
\dot{\widetilde{\delta}}=\omega-\dot{\delta}_{r}(t)
$$

As we want that the error system be a stable linear system, we state $\omega^{*}$ as the desired value for $\omega$ (taking $\lambda_{1}>0$ ):

$$
\omega^{*}=-\lambda_{1} \widetilde{\delta}+\dot{\delta_{r}}
$$

and then we may define:

$$
\widetilde{\omega} \triangleq \omega-\omega^{*}=\omega+\lambda_{1} \widetilde{\delta}-\dot{\delta}_{r}
$$

Taking the time derivatives of both equations leads to:

$$
\begin{aligned}
\dot{\tilde{\delta}} & =-\lambda_{1} \widetilde{\delta}+\widetilde{\omega} \\
\dot{\widetilde{\omega}} & =-\frac{D}{H} \omega+\frac{\omega_{s}}{H}\left(\theta(t)-P_{e}\right)-\lambda_{1}^{2} \widetilde{\delta}+\lambda_{1} \widetilde{\omega}-\ddot{\delta}_{r}
\end{aligned}
$$

Following the same technique, we define $\left(\lambda_{2}>0, k>0\right)$ the reference signal for $P_{e}$ that linearizes our system:

$$
P_{e}^{*}=\frac{H}{\omega_{s}}\left\{-\frac{D}{H} \omega-\lambda_{1}^{2} \widetilde{\delta}+\lambda_{1} \widetilde{\omega}-\ddot{\delta}_{r}+\lambda_{2} \widetilde{\omega}+\widetilde{\delta}+\frac{1}{4} k\left(\frac{\omega_{s}}{H}\right)^{2} \widetilde{\omega}\right\}+\hat{\theta}
$$

where $\hat{\theta}$ is an estimate of $\theta$ and

$$
\widetilde{P}_{e}=P_{e}-P_{e}^{*}
$$

Rewriting the second equation of (14):

Prepared using rncauth.cls

Int. J. Robust Nonlinear Control 20; :- 


$$
\dot{\tilde{\omega}}=\frac{\omega_{s}}{H} \widetilde{\theta}(t)-\frac{\omega_{s}}{H} \widetilde{P}_{e}-\lambda_{2} \widetilde{\omega}-\widetilde{\delta}-\frac{1}{4} k\left(\frac{\omega_{s}}{H}\right)^{2} \widetilde{\omega}
$$

and taking the derivative of $P_{e}^{*}$ :

$$
\begin{aligned}
\dot{P}_{e}^{*}= & \frac{H}{\omega_{s}}\left\{-\frac{D}{H}\left(\dot{\widetilde{\omega}}-\lambda_{1} \dot{\widetilde{\delta}}+\ddot{\delta}_{r}\right)\right. \\
& +\left(\lambda_{1}+\lambda_{2}+\frac{1}{4} k\left(\frac{\omega_{s}}{H}\right)^{2}\right)\left(-\frac{D}{H} \omega+\frac{\omega_{s}}{H}\left(\theta(t)-P_{e}\right)-\lambda_{1}^{2} \widetilde{\delta}+\lambda_{1} \widetilde{\omega}-\ddot{\delta}_{r}\right) \\
& \left.+\left(1-\lambda_{1}^{2}\right)\left(-\lambda_{1} \widetilde{\delta}+\widetilde{\omega}\right)\right\}+\dot{\hat{\theta}}-\frac{H}{\omega_{s}} \dot{\ddot{\delta}}_{r}
\end{aligned}
$$

equation (12) can finally be rewritten as $(\widetilde{\theta}=\theta-\hat{\theta})$

$$
\begin{aligned}
\dot{\widetilde{\delta}} & =-\lambda_{1} \widetilde{\delta}+\widetilde{\omega} \\
\dot{\tilde{\omega}} & =-\widetilde{\delta}-\lambda_{2} \widetilde{\omega}-\frac{\omega_{s}}{H} \widetilde{P}_{e}-\frac{k}{4}\left(\frac{\omega_{s}}{H}\right)^{2} \widetilde{\omega}+\frac{\omega_{s}}{H} \widetilde{\theta} \\
\dot{\widetilde{P}}_{e} & =-\frac{1}{T_{d 0}^{\prime}} P_{e}+\frac{V_{s}}{X_{d s} T_{d 0}^{\prime}} \sin (\delta) K_{c} u_{f}+\frac{\left(X_{d}-X_{d}^{\prime}\right) V_{s}^{2}}{X_{d s} X_{d s}^{\prime}} \omega \sin ^{2}(\delta)+P_{e} \omega \cot (\delta) \\
& -\frac{H}{\omega_{s}}\left\{\left(-\lambda_{1}^{2}+1+\lambda_{1} \frac{D}{H}\right)\left(-\lambda_{1} \widetilde{\delta}+\widetilde{\omega}\right)\right. \\
& \left.+\left(-\frac{D}{H}+\lambda_{1}+\lambda_{2}+\frac{k}{4}\left(\frac{\omega_{s}}{H}\right)^{2}\right)\left(-\frac{D}{H} \omega-\lambda_{1}^{2} \widetilde{\delta}+\lambda_{1} \widetilde{\omega}-\frac{\omega_{s}}{H} P_{e}-\ddot{\delta}_{r}\right)\right\} \\
& -\left(-\frac{D}{H}+\lambda_{1}+\lambda_{2}+\frac{k}{4}\left(\frac{\omega_{s}}{H}\right)^{2}\right) \hat{\theta}-\dot{\hat{\theta}} \\
& -\left(-\frac{D}{H}+\lambda_{1}+\lambda_{2}+\frac{k}{4}\left(\frac{\omega_{s}}{H}\right)^{2}\right) \widetilde{\theta}+\frac{D}{\omega_{s}} \ddot{\delta}_{r}+\frac{H}{\omega_{s}} \dot{\tilde{\delta}}_{r}
\end{aligned}
$$

We can see from equation (15) that in order to compute our control signal we need the derivatives of $\delta_{r}$. To do so, we must remember that:

$$
\begin{aligned}
& \dot{\delta}_{r}=\frac{d \delta_{r}}{d \widehat{P}_{m}} \frac{d \widehat{P}_{m}}{d t} \\
& \ddot{\delta}_{r}=\frac{d^{2} \delta_{r}}{d \widehat{P}_{m}^{2}} \frac{d \widehat{P}_{m}}{d t}+\frac{d \delta_{r}}{d \widehat{P}_{m}} \frac{d^{2} \widehat{P}_{m}}{d t^{2}} \\
& \ddot{\tilde{\delta}}_{r}=\frac{d^{3} \delta_{r}}{d \widehat{P}_{m}^{3}} \frac{d \widehat{P}_{m}}{d t}+2 \frac{d^{2} \delta_{r}}{d \widehat{P}_{m}^{2}} \frac{d^{2} \widehat{P}_{m}}{d t^{2}}+\frac{d \delta_{r}}{d \widehat{P}_{m}} \frac{d^{3} \widehat{P}_{m}}{d t^{3}}
\end{aligned}
$$

These computations may be seen in the Appendix, leading to:

Prepared using rncauth.cls

Int. J. Robust Nonlinear Control 20; :- 


$$
\begin{aligned}
\dot{\delta}_{r}= & \gamma_{1} \frac{d \delta_{r}}{d \widehat{P}_{m}} \widetilde{\omega}_{e} \\
\ddot{\delta}_{r}= & \gamma_{1} \frac{d^{2} \delta_{r}}{d \widehat{P}_{m}^{2}} \widetilde{\omega}_{e}+\gamma_{1} \frac{d \delta_{r}}{d \widehat{P}_{m}} \frac{\omega_{s}}{H}\left(\widehat{\theta}-\widehat{P}_{m}\right)-\gamma_{1} \frac{d \delta_{r}}{d \widehat{P}_{m}} \frac{D}{H} \widetilde{\omega}_{e}+\gamma_{1} \frac{d \delta_{r}}{d \widehat{P}_{m}} \frac{\omega_{s}}{H} \widetilde{\theta} \\
\dot{\tilde{\delta}}_{r}= & \gamma_{1} \frac{d^{3} \delta_{r}}{d \widehat{P}_{m}^{3}} \widetilde{\omega}_{e}+2 \gamma_{1} \frac{d^{2} \delta_{r}}{d \widehat{P}_{m}^{2}} \frac{\omega_{s}}{H}\left(\widehat{\theta}-\widehat{P}_{m}\right)-2 \gamma_{1} \frac{d^{2} \delta_{r}}{d \widehat{P}_{m}^{2}} \frac{D}{H} \widetilde{\omega}_{e}+2 \gamma_{1} \frac{d^{2} \delta_{r}}{d \widehat{P}_{m}^{2}} \frac{\omega_{s}}{H} \widetilde{\theta} \\
& +\left(\gamma_{1} \frac{D^{2}}{H^{2}}-\gamma_{1}^{2} \frac{\omega_{s}}{H}\right) \frac{d \delta_{r}}{d \widehat{P}_{m}} \widetilde{\omega}_{e}-\gamma_{1} \frac{d \delta_{r}}{d \widehat{P}_{m}} \frac{D \omega_{s}}{H^{2}}\left(\widehat{\theta}-\widehat{P}_{m}\right)-\gamma_{1} \frac{d \delta_{r}}{d \widehat{P}_{m}} \frac{D \omega_{s}}{H^{2}} \widetilde{\theta}
\end{aligned}
$$

where $\frac{d \delta_{r}}{d \widehat{P}_{m}}, \frac{d^{2} \delta_{r}}{d \widehat{P}_{m}^{2}}$ and $\frac{d^{3} \delta_{r}}{d \widehat{P}_{m}^{3}}$ are given by (26), (28) and (30) in the Appendix.

Because some of the terms of $\ddot{\delta}_{r}$ and $\dot{\tilde{\delta}}_{r}$ are not available for feedback, we define new variables $\ddot{\delta}_{r u}$ and $\dot{\tilde{\delta}}_{r u}$ that will be used for our control law. These variables are defined such that:

$$
\begin{aligned}
& \dot{\delta}_{r}-\dot{\delta}_{r u}=0 \\
& \ddot{\delta}_{r}-\ddot{\delta}_{r u}=\gamma_{1} \frac{d \delta_{r}}{d \widehat{P}_{m}} \frac{\omega_{s}}{H} \widetilde{\theta} \\
& \dot{\tilde{\delta}}_{r}-\ddot{\tilde{\delta}}_{r u}=2 \gamma_{1} \frac{d^{2} \delta_{r}}{d \widehat{P}_{m}^{2}} \frac{\omega_{s}}{H} \widetilde{\theta}-\gamma_{1} \frac{d \delta_{r}}{d \widehat{P}_{m}} \frac{D \omega_{s}}{H^{2}} \widetilde{\theta}
\end{aligned}
$$

Defining $\left(\lambda_{3}>0\right)$, we may compute the control signal that will linearize the last equation of (15):

$$
\begin{aligned}
u_{f} & =\frac{T_{d 0}^{\prime} X_{d s}}{V_{s} K_{c} \sin (\delta)} \phi_{0} \\
\phi_{0} & =\frac{1}{T_{d 0}^{\prime}} P_{e}-\frac{\left(X_{d}-X_{d}^{\prime}\right)}{X_{d s} X_{d s}^{\prime}} V_{s}^{2} \omega \sin ^{2}(\delta)-P_{e} \omega \cot (\delta) \\
& +\frac{H}{\omega_{s}}\left\{\left(-\lambda_{1}^{2}+1+\lambda_{1} \frac{D}{H}\right)\left(-\lambda_{1} \widetilde{\delta}+\widetilde{\omega}\right)\right. \\
& \left.+\left(-\frac{D}{H}+\lambda_{1}+\lambda_{2}+\frac{k}{4}\left(\frac{\omega_{s}}{H}\right)^{2}\right)\left(-\frac{D}{H} \omega-\lambda_{1}^{2} \widetilde{\delta}+\lambda_{1} \widetilde{\omega}-\frac{\omega_{s}}{H} P_{e}-\ddot{\delta}_{r u}\right)\right\} \\
& +\left(-\frac{D}{H}+\lambda_{1}+\lambda_{2}+\frac{k}{4}\left(\frac{\omega_{s}}{H}\right)^{2}\right) \hat{\theta}+\dot{\hat{\theta}} \\
& -\frac{k}{4}\left(-\frac{D}{H}+\lambda_{1}+\lambda_{2}+\frac{k}{4}\left(\frac{\omega_{s}}{H}\right)^{2}\right)^{2} \widetilde{P}_{e}-\lambda_{3} \widetilde{P}_{e}+\frac{\omega_{s}}{H} \widetilde{\omega}-\frac{D}{\omega_{s}} \ddot{\delta}_{r u}-\frac{H}{\omega_{s}} \dot{\tilde{\delta}}_{r u}
\end{aligned}
$$

Remark here the use of $\delta_{r u}$ as the feedback available variable. Now, defining the new constant:

$$
c_{1} \triangleq\left(-\frac{D}{H}+\lambda_{1}+\lambda_{2}+\frac{k}{4}\left(\frac{\omega_{s}}{H}\right)^{2}\right)
$$

Prepared using rncauth.cls

Int. J. Robust Nonlinear Control 20; :- 
we may rewrite the previous equations as:

$$
\begin{aligned}
u_{f}= & \frac{T_{d 0}^{\prime} X_{d s}}{V_{s} K_{c} \sin (\delta)} \phi_{0} \\
\phi_{0}= & \frac{1}{T_{d 0}^{\prime}} P_{e}-\frac{\left(X_{d}-X_{d}^{\prime}\right)}{X_{d s} X_{d s}^{\prime}} V_{s}^{2} \omega \sin ^{2}(\delta)-P_{e} \omega \cot (\delta)+c_{1} \hat{\theta}+\dot{\hat{\theta}} \\
+ & \frac{H}{\omega_{s}}\left\{\left(-\lambda_{1}^{2}+1+\lambda_{1} \frac{D}{H}\right)\left(-\lambda_{1} \widetilde{\delta}+\widetilde{\omega}\right)+c_{1}\left(-\frac{D}{H} \omega-\lambda_{1}^{2} \widetilde{\delta}+\lambda_{1} \widetilde{\omega}-\frac{\omega_{s}}{H} P_{e}-\ddot{\delta}_{r u}\right)\right\} \\
& -\frac{k}{4}\left(c_{1}-\gamma_{1} c_{1} \frac{d \delta_{r}}{d \widehat{P}_{m}}-2 \gamma_{1} \frac{d^{2} \delta_{r}}{d \widehat{P}_{m}^{2}}\right)^{2} \widetilde{P}_{e}-\lambda_{3} \widetilde{P}_{e}+\frac{\omega_{s}}{H} \widetilde{\omega}-\frac{D}{\omega_{s}} \ddot{\delta}_{r u}-\frac{H}{\omega_{s}} \dot{\ddot{\delta}_{r u}}
\end{aligned}
$$

and:

$$
\begin{aligned}
\dot{\widetilde{P}}_{e}= & -\frac{1}{T_{d 0}^{\prime}} P_{e}+\frac{\left(X_{d}-X_{d}^{\prime}\right) V_{s}^{2}}{X_{d s} X_{d s}^{\prime}} \omega \sin ^{2}(\delta)+P_{e} \omega \cot (\delta) \\
& -\frac{H}{\omega_{s}}\left\{\left(-\lambda_{1}^{2}+1+\lambda_{1} \frac{D}{H}\right)\left(-\lambda_{1} \widetilde{\delta}+\widetilde{\omega}\right)+c_{1}\left(-\frac{D}{H} \omega-\lambda_{1}^{2} \widetilde{\delta}+\lambda_{1} \widetilde{\omega}-\frac{\omega_{s}}{H} P_{e}-\ddot{\delta}_{r}\right)\right\} \\
& -c_{1} \hat{\theta}-\dot{\hat{\theta}}-c_{1} \widetilde{\theta}+\frac{D}{\omega_{s}} \ddot{\delta}_{r}+\frac{H}{\omega_{s}} \dot{\ddot{\delta}}_{r}+\frac{V_{s}}{X_{d s} T_{d 0}^{\prime}} \sin (\delta) K_{c} \frac{T_{d 0}^{\prime} X_{d s}}{V_{s} K_{c} \sin (\delta)} \phi_{0}
\end{aligned}
$$

Substituting (17) in (18) one will find:

$$
\begin{aligned}
\dot{\tilde{P}}_{e}= & -\frac{1}{T_{d 0}^{\prime}} P_{e}+\frac{\left(X_{d}-X_{d}^{\prime}\right) V_{s}^{2}}{X_{d s} X_{d s}^{\prime}} \omega \sin ^{2}(\delta)+P_{e} \omega \cot (\delta) \\
& -\frac{H}{\omega_{s}}\left\{\left(-\lambda_{1}^{2}+1+\lambda_{1} \frac{D}{H}\right)\left(-\lambda_{1} \widetilde{\delta}+\widetilde{\omega}\right)+c_{1}\left(-\frac{D}{H} \omega-\lambda_{1}^{2} \widetilde{\delta}+\lambda_{1} \widetilde{\omega}-\frac{\omega_{s}}{H} P_{e}-\ddot{\delta}_{r}\right)\right\} \\
& -c_{1} \hat{\theta}-\dot{\hat{\theta}}-c_{1} \widetilde{\theta}+\frac{D}{\omega_{s}} \ddot{\delta}_{r}+\frac{H}{\omega_{s}} \ddot{\tilde{\delta}}_{r} \\
& +\frac{1}{T_{d 0}^{\prime}} P_{e}-\frac{\left(X_{d}-X_{d}^{\prime}\right)}{X_{d s} X_{d s}^{\prime}} V_{s}^{2} \omega \sin ^{2}(\delta)-P_{e} \omega \cot (\delta) \\
& +\frac{H}{\omega_{s}}\left\{\left(-\lambda_{1}^{2}+1+\lambda_{1} \frac{D}{H}\right)\left(-\lambda_{1} \widetilde{\delta}+\widetilde{\omega}\right)+c_{1}\left(-\frac{D}{H} \omega-\lambda_{1}^{2} \widetilde{\delta}+\lambda_{1} \widetilde{\omega}-\frac{\omega_{s}}{H} P_{e}-\ddot{\delta}_{r u}\right)\right\} \\
& +c_{1} \hat{\theta}+\dot{\hat{\theta}}-\frac{k}{4}\left(c_{1}-\gamma_{1} c_{1} \frac{d \delta_{r}}{d \widehat{P}_{m}}-2 \gamma_{1} \frac{d^{2} \delta_{r}}{d \widehat{P}_{m}^{2}}\right)^{2} \widetilde{P}_{e}-\lambda_{3} \widetilde{P}_{e}+\frac{\omega_{s}}{H} \widetilde{\omega}-\frac{D}{\omega_{s}} \ddot{\delta}_{r u}-\frac{H}{\omega_{s}} \dot{\tilde{\delta}}_{r u}
\end{aligned}
$$

that may be rewritten as:

$$
\dot{\widetilde{P}}_{e}=-\frac{k}{4}\left(c_{1}-\gamma_{1} c_{1} \frac{d \delta_{r}}{d \widehat{P}_{m}}-2 \gamma_{1} \frac{d^{2} \delta_{r}}{d \widehat{P}_{m}^{2}}\right)^{2} \widetilde{P}_{e}-\lambda_{3} \widetilde{P}_{e}+\frac{\omega_{s}}{H} \widetilde{\omega}-\left(c_{1}-\gamma_{1} c_{1} \frac{d \delta_{r}}{d \widehat{P}_{m}}-2 \gamma_{1} \frac{d^{2} \delta_{r}}{d \widehat{P}_{m}^{2}}\right) \widetilde{\theta}
$$

Then, the closed loop system becomes 


$$
\begin{aligned}
\dot{\tilde{\delta}}= & -\lambda_{1} \tilde{\delta}+\tilde{\omega} \\
\dot{\tilde{\omega}}= & -\tilde{\delta}-\lambda_{2} \tilde{\omega}-\frac{\omega_{s}}{H} \widetilde{P}_{e}-\frac{k}{4}\left(\frac{\omega_{s}}{H}\right)^{2} \tilde{\omega}+\frac{\omega_{s}}{H} \tilde{\theta} \\
\dot{\widetilde{P}}_{e}= & -\frac{k}{4}\left(c_{1}-\gamma_{1} c_{1} \frac{d \delta_{r}}{d \widehat{P}_{m}}-2 \gamma_{1} \frac{d^{2} \delta_{r}}{d \widehat{P}_{m}^{2}}\right)^{2} \widetilde{P}_{e} \\
& -\lambda_{3} \widetilde{P}_{e}+\frac{\omega_{s}}{H} \widetilde{\omega}-\left(c_{1}-\gamma_{1} c_{1} \frac{d \delta_{r}}{d \widehat{P}_{m}}-2 \gamma_{1} \frac{d^{2} \delta_{r}}{d \widehat{P}_{m}^{2}}\right) \tilde{\theta}
\end{aligned}
$$

The adaptation law is ( $\gamma$ is a positive adaptation gain)

$$
\dot{\hat{\theta}}=\gamma \operatorname{Proj}\left(\left(-\widetilde{P}_{e}\left(c_{1}-\gamma_{1} c_{1} \frac{d \delta_{r}}{d \widehat{P}_{m}}-2 \gamma_{1} \frac{d^{2} \delta_{r}}{d \widehat{P}_{m}^{2}}\right)+\widetilde{\omega} \frac{\omega_{s}}{H}\right), \hat{\theta}\right)
$$

where $\operatorname{Proj}(y, \hat{\theta})$ is the smooth projection algorithm introduced in [16]

$$
\begin{array}{llll}
\operatorname{Proj}(y, \hat{\theta}) & =y, & & \text { if } p(\hat{\theta}) \leq 0 \\
\operatorname{Proj}(y, \hat{\theta}) & =y, & & \text { if } p(\hat{\theta}) \geq 0 \text { and }\langle\operatorname{gradp}(\hat{\theta}), y\rangle \leq 0 \\
\operatorname{Proj}(y, \hat{\theta}) & =[1-p(\hat{\theta})|\operatorname{grad} p(\hat{\theta})|], & \text { otherwise }
\end{array}
$$

with

$$
p(\theta)=\frac{\left(\theta-\frac{\theta_{M}+\theta_{m}}{2}\right)^{2}-\left(\frac{\theta_{M}-\theta_{m}}{2}\right)}{\epsilon^{2}+2 \epsilon\left(\frac{\theta_{M}-\theta_{m}}{2}\right)}
$$

for $\epsilon$ an arbitrary positive constant, which guarantees in particular that:

$$
\begin{array}{cl}
\text { i) } & \theta_{m}-\epsilon \leq \hat{\theta}(t) \leq \theta_{M}+\epsilon \\
\text { ii) } & |\operatorname{Proj}(y, \hat{\theta})| \leq|y| \\
\text { iii) } & (\theta-\hat{\theta}) \operatorname{Proj}(y, \hat{\theta}) \geq(\theta-\hat{\theta}) y
\end{array}
$$

We may remark that if $\widetilde{P_{e}}$ and $\widetilde{\omega}$ were the errors from the state to an equilibrium point, the adaptation law would be equivalent to a gradient approach. But this analysis, globally, is not true since these two errors signals are not the state errors. Only in a small region around the equilibrium point this would be valid.

To compute this adaptation law, let's consider the function:

$$
W=\frac{1}{2}\left(\widetilde{\delta}^{2}+\widetilde{\omega}^{2}+\widetilde{P}_{e}^{2}\right)
$$

whose time derivative, according to (19), is

Prepared using rncauth.cls

Int. J. Robust Nonlinear Control 20; :- 


$$
\begin{aligned}
\dot{W}= & -\lambda_{1} \widetilde{\delta}^{2}-\lambda_{2} \widetilde{\omega}^{2}-\lambda_{3} \widetilde{P}_{e}^{2}+\widetilde{\omega} \frac{\omega_{s}}{H} \widetilde{\theta}-\frac{k}{4}\left(\frac{\omega_{s}}{H}\right)^{2} \widetilde{\omega}^{2} \\
& -\frac{k}{4}\left(c_{1}-\gamma_{1} c_{1} \frac{d \delta_{r}}{d \widehat{P}_{m}}-2 \gamma_{1} \frac{d^{2} \delta_{r}}{d \widehat{P}_{m}^{2}}\right)^{2} \widetilde{P}_{e}^{2}-\left(c_{1}-\gamma_{1} c_{1} \frac{d \delta_{r}}{d \widehat{P}_{m}}-2 \gamma_{1} \frac{d^{2} \delta_{r}}{d \widehat{P}_{m}^{2}}\right) \widetilde{\theta} \widetilde{P}_{e}
\end{aligned}
$$

Completing the squares, we obtain the inequality

$$
\dot{W} \leq-\lambda_{1} \widetilde{\delta}^{2}-\lambda_{2} \widetilde{\omega}^{2}-\lambda_{3} \widetilde{P}_{e}^{2}+\frac{2}{k} \widetilde{\theta}^{2}
$$

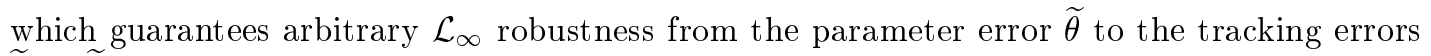
$\widetilde{\delta}, \widetilde{\omega}, \widetilde{P}_{e}$ (see [9] Section 5.4).

The projection algorithms (21) guarantee that $\tilde{\theta}$ is bounded, and, by virtue of (22) and (23), that $\widetilde{\delta}, \widetilde{\omega}$ and $\widetilde{P}_{e}$ are bounded. Therefore, $\dot{\hat{\theta}}$ is bounded. Integrating (23), we have for every $t \geq t_{0} \geq 0$

$$
-\int_{t_{0}}^{t}\left(\lambda_{1} \widetilde{\delta}^{2}+\lambda_{2} \widetilde{\omega}^{2}+\lambda_{3} \widetilde{P}_{e}^{2}\right) d \tau+\frac{2}{k} \int_{t_{0}}^{t} \widetilde{\theta}^{2} d \tau \geq W(t)-W\left(t_{0}\right)
$$

Since $W(t) \geq 0$ and, by virtue of the projection algorithm (21),

$$
\widetilde{\theta}(t) \leq \theta_{M}-\theta_{m}+\epsilon
$$

it follows that

$$
\int_{t_{0}}^{t}\left(\lambda_{1} \widetilde{\delta}^{2}+\lambda_{2} \widetilde{\omega}^{2}+\lambda_{3} \widetilde{P}_{e}^{2}\right) d \tau \leq W\left(t_{0}\right)+\frac{2}{k}\left(\theta_{M}-\theta_{m}+\epsilon\right)^{2}\left(t-t_{0}\right)
$$

which, if $W\left(t_{0}\right)=0$ (i.e. $t_{0}$ is a time before the occurrence of the fault), implies arbitrary $\mathcal{L}_{2}$ attenuation (by a factor $k$ ) of the errors $\widetilde{\delta}, \widetilde{\omega}$ and $\widetilde{P}_{e}$ caused by the fault. To analyze the asymptotic behavior of the adaptive control, we consider the function

Its time derivative is:

$$
V=\frac{1}{2}\left(\widetilde{\delta}^{2}+\widetilde{\omega}^{2}+\widetilde{P}_{e}^{2}\right)+\frac{1}{2} \frac{1}{\gamma} \widetilde{\theta}^{2}
$$

$$
\begin{aligned}
\dot{V} & =-\lambda_{1} \widetilde{\delta}^{2}-\lambda_{2} \widetilde{\omega}^{2}-\lambda_{3} \widetilde{P}_{e}^{2}+\widetilde{\omega} \frac{\omega_{s}}{H} \widetilde{\theta}-\frac{k}{4}\left(\frac{\omega_{s}}{H}\right)^{2} \widetilde{\omega}^{2}+\frac{1}{\gamma} \tilde{\theta} \tilde{\theta} \\
& -\left(c_{1}-\gamma_{1} c_{1} \frac{d \delta_{r}}{d \widehat{P}_{m}}-2 \gamma_{1} \frac{d^{2} \delta_{r}}{d \widehat{P}_{m}^{2}}\right) \widetilde{\theta} \widetilde{P}_{e}-\frac{k}{4}\left(c_{1}-\gamma_{1} c_{1} \frac{d \delta_{r}}{d \widehat{P}_{m}}-2 \gamma_{1} \frac{d^{2} \delta_{r}}{d \widehat{P}_{m}^{2}}\right)^{2} \widetilde{P}_{e}^{2}
\end{aligned}
$$

Then, using the adaptation law, we may find (remember that $(\dot{\widetilde{\theta}}=-\dot{\hat{\theta}})$ ):

$$
\dot{V}=-\lambda_{1} \widetilde{\delta}^{2}-\lambda_{2} \widetilde{\omega}^{2}-\lambda_{3} \widetilde{P}_{e}^{2}-\frac{k}{4}\left(\frac{\omega_{s}}{H}\right)^{2} \widetilde{\omega}^{2}-\frac{k}{4}\left(c_{1}-\gamma_{1} c_{1} \frac{d \delta_{r}}{d \widehat{P}_{m}}-2 \gamma_{1} \frac{d^{2} \delta_{r}}{d \widehat{P}_{m}^{2}}\right)^{2} \widetilde{P}_{e}^{2}
$$


The projection estimation algorithm (21) is designed so that the time derivative of $\mathrm{V}$ satisfies

$$
\dot{V} \leq-\lambda_{1} \widetilde{\delta}^{2}-\lambda_{2} \widetilde{\omega}^{2}-\lambda_{3} \widetilde{P}_{e}^{2}
$$

Integrating (24), we have

$$
\lim _{t \rightarrow \infty} \int_{t_{0}}^{t}\left(\lambda_{1} \widetilde{\delta}^{2}+\lambda_{2} \widetilde{\omega}^{2}+\lambda_{3} \widetilde{P}_{e}^{2}\right) d \tau \leq V(0)-V(\infty)<\infty
$$

From the boundedness of $\dot{\widetilde{\delta}}, \dot{\tilde{\omega}}$ and $\dot{\widetilde{P}}_{e}$, and Barbalat's Lemma (see [15], [13] and [17]) it follows that

$$
\lim _{t \rightarrow \infty}\left\|\left[\begin{array}{c}
\widetilde{\delta}(t) \\
\widetilde{\omega}(t) \\
\widetilde{P}_{e}(t)
\end{array}\right]\right\|=0
$$

We may now rewrite the closed loop system following the normal form:

$$
\begin{aligned}
\dot{\tilde{x}} & =A \widetilde{x}+\Omega^{T} \widetilde{\theta} \\
\dot{\widetilde{\theta}} & =-\Lambda \Omega \widetilde{x}
\end{aligned}
$$

which leads to:

$$
\begin{aligned}
\dot{\tilde{x}}= & {\left[\begin{array}{ccc}
-\lambda_{1} & 1 & 0 \\
-1 & -\left(\lambda_{2}+\frac{k}{4}\left(\frac{\omega_{s}}{H}\right)^{2}\right) & -\frac{\omega_{s}}{H} \\
0 & \frac{\omega_{s}}{H} & -\left(\lambda_{3}+\frac{k}{4}\left(-\frac{D}{H}+\lambda_{1}+\lambda_{2}+\frac{k}{4}\left(\frac{\omega_{s}}{H}\right)^{2}\right)^{2}\right)
\end{array}\right] \widetilde{x} } \\
& +\left[\begin{array}{c}
0 \\
-\left(-\frac{\omega_{s}}{H}+\lambda_{1}+\lambda_{2}+\frac{k}{4}\left(\frac{\omega_{s}}{H}\right)^{2}\right)
\end{array}\right] \widetilde{\theta} \\
\dot{\tilde{\theta}}= & -\gamma\left[\begin{array}{lll}
0 & \frac{\omega_{s}}{H} & \left.\left(\frac{D}{H}-\lambda_{1}-\lambda_{2}-\frac{k}{4}\left(\frac{\omega_{s}}{H}\right)^{2}\right)\right] \widetilde{x}
\end{array}\right.
\end{aligned}
$$

And then computing (for a constant $c_{2}$ ):

$$
\Omega \Omega^{T}=\frac{\omega_{s}^{2}}{H^{2}}+\left(-\frac{D}{H}+\lambda_{1}+\lambda_{2}+\frac{k}{4}\left(\frac{\omega_{s}}{H}\right)^{2}\right)^{2} \triangleq c_{2}>0
$$

we then may show by persistency of excitation (see [13], [17] and [15]) that $\widetilde{x}$ and $\widetilde{\theta}$ will be globally exponentially stable, and then all error signals go exponentially to zero, for all (at least) $C^{3} \delta_{r}\left(\widehat{P}_{m}, x\right)$.

It is important to remark a very interesting feature of the proposed controller: all states go exponentially to the faulted equilibrium point that is completely unknown. Actually, all states go exponentially to trajectories that go themselves exponentially to the unknown equilibrium 
point. We must also remark that both convergencies are simultaneous. To detail this feature, note that $\delta$ will converge to the trajectory $\delta_{r}$ but, since $\delta_{r}$ is a one-to-one smooth function of $\widehat{P}_{m}$, it will converge exponentially to the correct equilibrium value $\delta_{s}$ as $\widehat{P}_{m}$ converges exponentially to $P_{m}$. This means that the reference trajectory $\left(\delta_{r}\right)$ will converge exponentially to the unknown equilibrium point $\left(\delta_{s}\right)$, and this convergence will be simultaneous to the convergence of the power angle $(\delta)$ to the trajectory $\left(\delta_{r}\right)$, what implies that $\lim _{t \rightarrow \infty}\left(\delta-\delta_{s}\right)=0$ exponentially. We must remark that the same happens to the other states ( $\omega$ and $P_{e}$ ). They converge to their reference trajectories $\left[\omega^{*}, P_{e}^{*}\right]$, and these trajectories converge to the faulted (unknown for $P_{e}$ ) equilibrium points of $\omega$ and $P_{e}$ as $\widetilde{\omega}(t)$ and $\widetilde{P}_{e}(t)$ converge to zero.

\section{Remark}

We must observe that there are two adapted values for the mechanical power. The reason is that even if both results finally recover the same value, they are not used for the same purpose, neither as equivalent variables. Note that $\widehat{P}_{m}$ is the estimation of the unknown parameter $P_{m}$, replacing it in the process of building the trajectories. It was designed purposely as an estimator and its behavior can be defined as desired, such that it can respect the restrictions imposed for our trajectories, mainly with respect to being at least $C^{3}$. Furthermore, its time derivatives, that are needed for the controller, are available. As a consequence, $\widehat{P}_{m}$ is very well behaved, going smoothly to the correct value of $P_{m}$.

On the other hand, $\widehat{\theta}$ was designed as the control adaptation. Even if it finally recovers the correct value of $P_{m}$ (faster than $\widehat{P}_{m}$ in some cases), it is not as well behaved, nor its time derivatives are available. As a control variable, it was expected to be swift. That is what assures the awareness of the control signal, being able to act very fast to assure the stability of the power generator.

\section{Simulation Results}

In this section we present simulations of the proposed controller, using the following data:

$$
\begin{array}{ccc}
\omega_{s}=314.159 \mathrm{rad} / \mathrm{s} & D=5 \mathrm{p} . \mathrm{u} . & H=8 \mathrm{~s} \\
T_{d 0}=6.9 \mathrm{~s} & K_{c}=1 & X_{d}=1.863 \mathrm{p} . \mathrm{u} . \\
X_{d}^{\prime}=0.257 \text { p.u. } & X_{T}=0.127 \text { p.u. } & X_{L}=0.4853 \text { p.u. }
\end{array}
$$

The operating point is $\delta_{s}=72^{\circ}, P_{m}=0.9$ p.u., $\omega_{0}=0$ to which corresponds $V_{t}=1$ p.u., with $V_{s}=1$ p.u..

The goal of the first simulation was to verify the effect of a severe fault on the turbine. It was considered a fast reduction of the mechanical input power, and the simulation was done according to the following sequence:

1. The system is in pre-faulted state.

2. At $t=0.5 s$ the mechanical input power begins to decrease.

3. At $t=5.5 \mathrm{~s}$ the mechanical input power is $50 \%$ of the initial value. 
The simulations were carried out using as control parameters:

$$
\begin{array}{ccc}
\lambda_{1}=2 & \lambda_{2}=10 & \lambda_{3}=100 \\
\gamma=0.1 & k=0.01 & \gamma_{1}=\frac{3 D^{2}}{4 H \omega_{s}}
\end{array}
$$

(a)

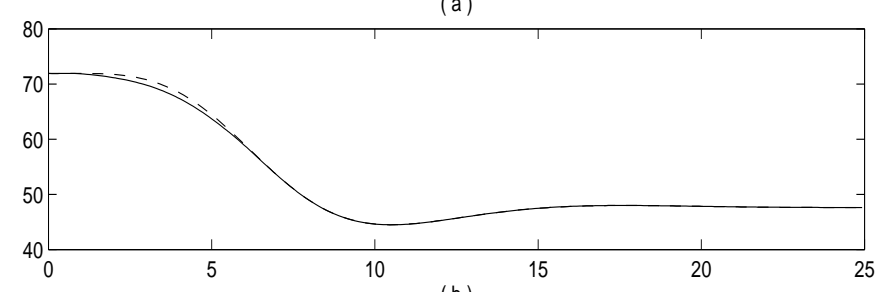

(b)
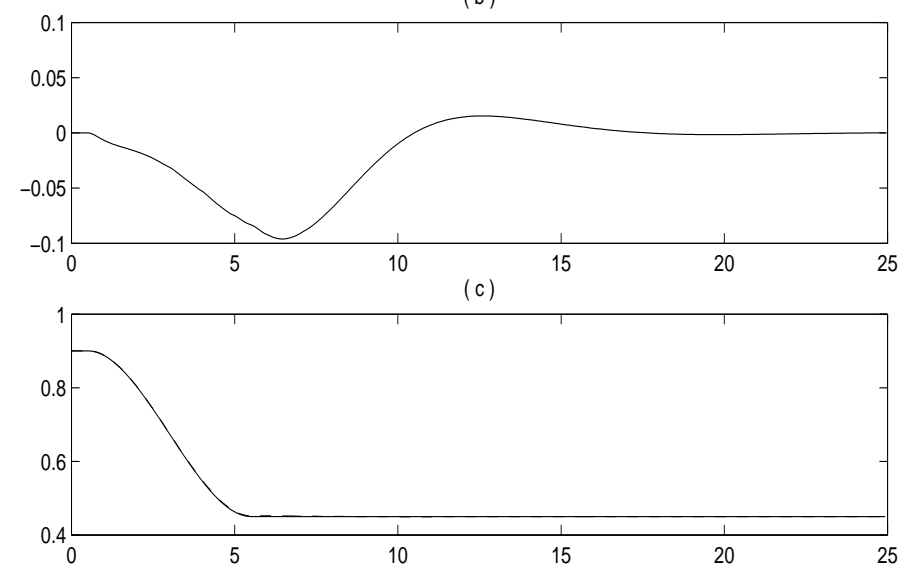

Figure 1. a) $\delta(-), \delta_{r}(--)$ b) $\omega$ c) $P_{e}(--), P_{m}(-)$

Fig. 1.a) shows that the trajectory for the power angle $\left(\delta_{r}\right)$ goes smoothly to its final $\operatorname{value}\left(\delta_{s}\right)$, and that $\delta$ matches it almost perfectly, being driven to its faulted unknown equilibrium point.

In Fig. 1.b) we see that the rotor velocity is correctly and smoothly driven to its equilibrium value, as well as the electrical power, driven to its trajectory that finally recovers the unknown equilibrium value as we may remark in Fig. 1.c)

Fig. 2.1a) shows how the output voltage drops during the fault, and goes to its correct value when the system is driven to the correct equilibrium point. If the estimation were not correct, there would be a steady state error.

One can see in Fig. 2.1b) that the control signal is very smooth and is kept inside the prescribed bounds.

We may see in Fig. 2.2a) the adapted value, $\hat{\theta}$, (dashed line) of the mechanical power (full line). It is accurate and swift, such that the correct value is adapted almost at once. We may remark that it recovers the correct value faster than the estimator does, as we may see in Fig. $2.2 \mathrm{~b}$ ) where it is plotted the estimated $\widehat{P}_{m}$ (dashed line) and the mechanical power (full line). 

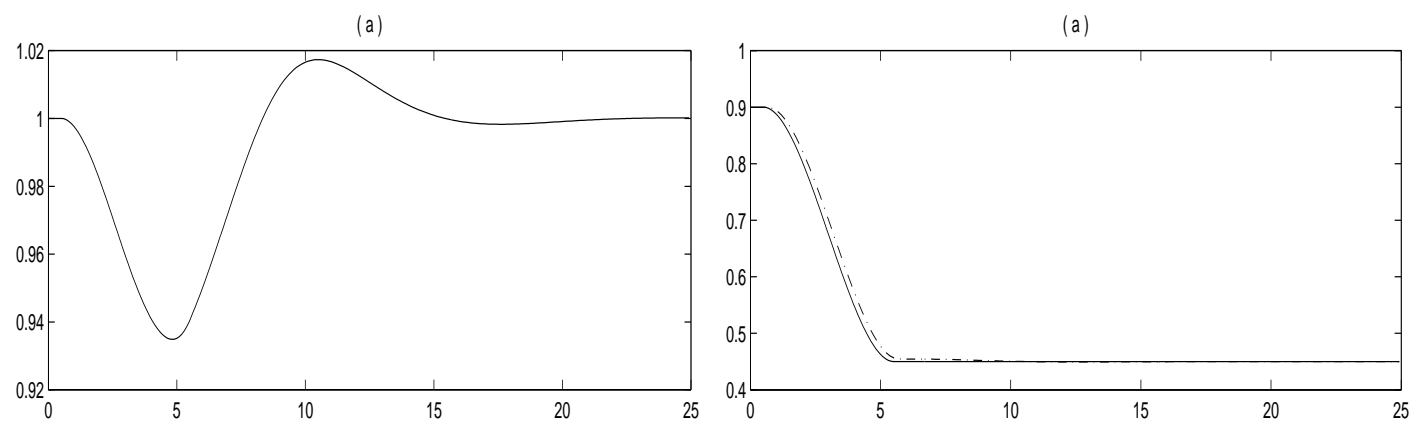

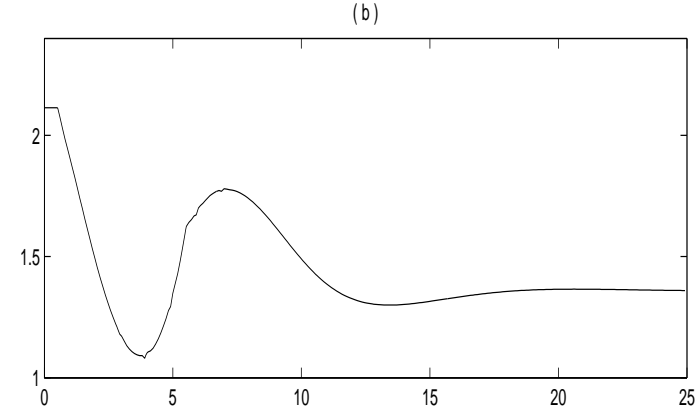

(1)

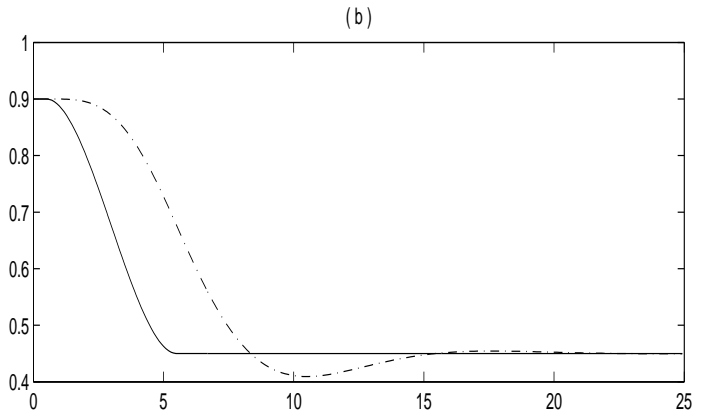

(2)

Figure 2. 1a) $V_{t}$ 1b) Control signal 2a) $\hat{\theta}(-.) P_{m}(-)$ 2b) $\widehat{P}_{m}(-),. P_{m}(-)$

On the other hand, the estimated value $\widehat{P}_{m}$ is very smooth, respecting the restrictions on the derivatives imposed for our tracked trajectory.

Note that during all time, the errors are very small. They can be made even smaller by increasing the parameter $k$. The choice of parameters is mainly based on the limitation of the control signal, as well as the desired bounds for states and outputs.

We present now the effect of faults on the transmission line. It was considered a large increment of line impedance, followed by a almost as large reduction. This is equivalent to the lost of part of the transmission lines, followed by a partial recover. Simulations were carried out following the sequence:

1. The system is in pre-faulted state.

2. At $t=1 s$ part of the power lines falls. This is reflected by an increment of line impedance in $33 \%$. Note that the change is instantaneous.

3. At $t=5 s$ part of the lines are recovered. This is seen as a reduction of $25 \%$ of the initial value of the line impedance.

The control parameters used for the simulations in this case are:

$$
\begin{array}{ccc}
\lambda_{1}=2 & \lambda_{2}=10 & \lambda_{3}=100 \\
\gamma=0.1 & k=0.01 & \gamma_{1}=\frac{3 D^{2}}{4 H \omega_{s}}
\end{array}
$$


(a)

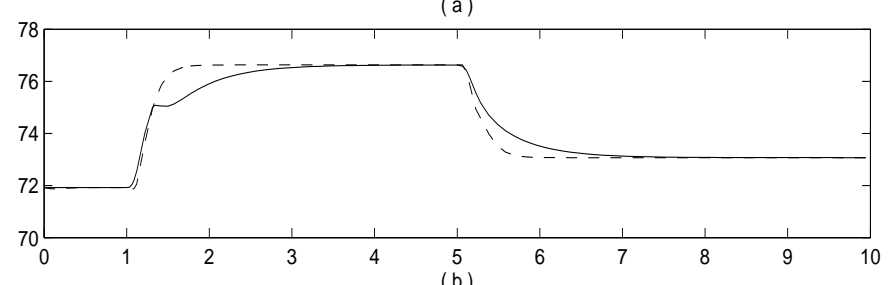

(b)
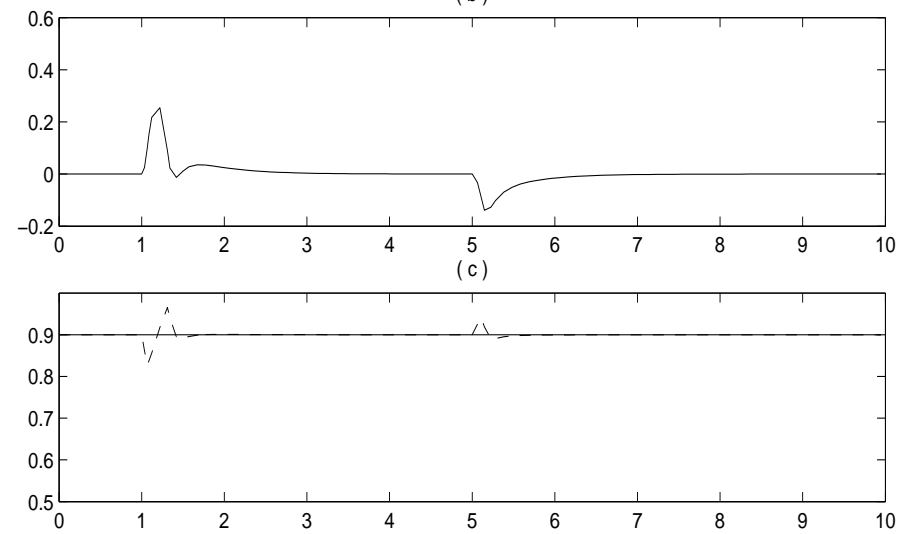

Figure 3. a) $\delta(-), \delta_{r}(--)$ b) $\omega$ c) $P_{e}(--), P_{m}(-)$

Fig. 3.a) shows that the trajectory (dashed line) for the power angle $\left(\delta_{r}\right)$ goes smoothly to its final value $\left(\delta_{s}\right)$, and that $\delta$ (full line) is able to track this trajectory, such that it is driven to its faulted equilibrium point.

In Fig. 3.b) and 3.c) we may see the other two states, the rotor velocity and the electrical power, being disturbed by the faults and then driven to their correct values by the controller. The same is verified in Fig. 4.1a) for the output voltage.

One can see in Fig. 4.1b) that the control signal is very fast, acting at once to keep the stability of our system. It is able to keep all signals inside the prescribed bounds, and to drive them to their correct values. Contrariwise the previous simulation where, as a mechanical fault, the perturbation was quite slow, here we see an electrical fault, then a much faster one, asking for a sharp response from the controller.

We may observe in Fig. 4.2a) the control adaptation variable $\hat{\theta}$ (dashed line) and the mechanical power (full line). In Fig. 4.2b), it is presented the estimation $\widehat{P}_{m}$ (dashed line) of the mechanical power $P_{m}$ (full line). One may then remark that both variables recover the same final value, but while $\widehat{P}_{m}$ keeps unchanged, $\hat{\theta}$ changes in time. This shows the difference between $\hat{\theta}$, as control variable, and $\widehat{P}_{m}$ as estimated value.

Finally, in Fig. 4.2c), one may see that the correct value for the transmission line impedance is computed by our technique. The value is recovered very fast, such that the system may be driven to its correct equilibrium point. This computation is filtered in order to respect physical limitations on the control signal magnitude. 

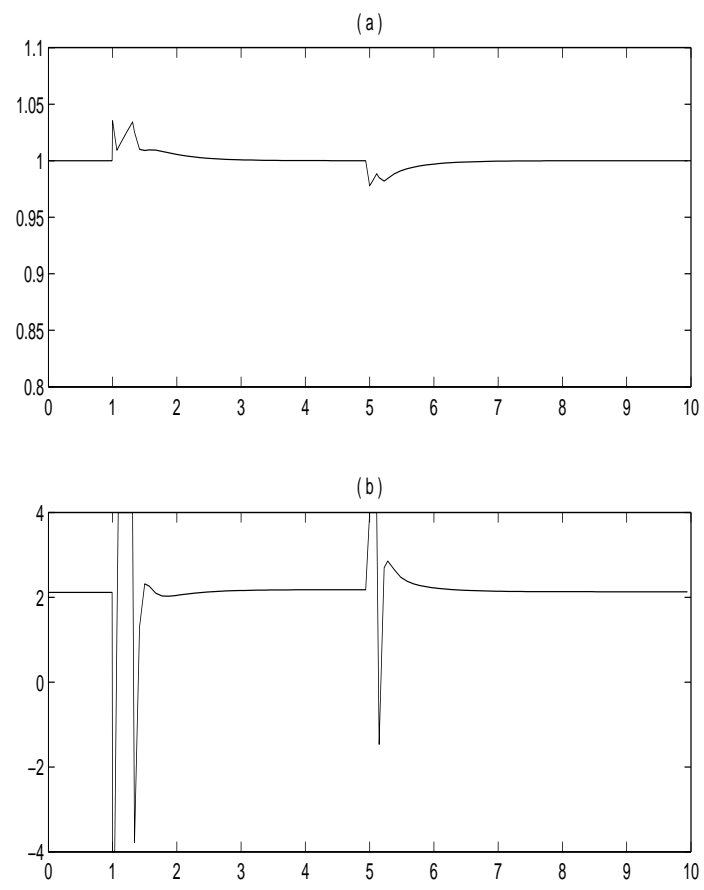

(1)
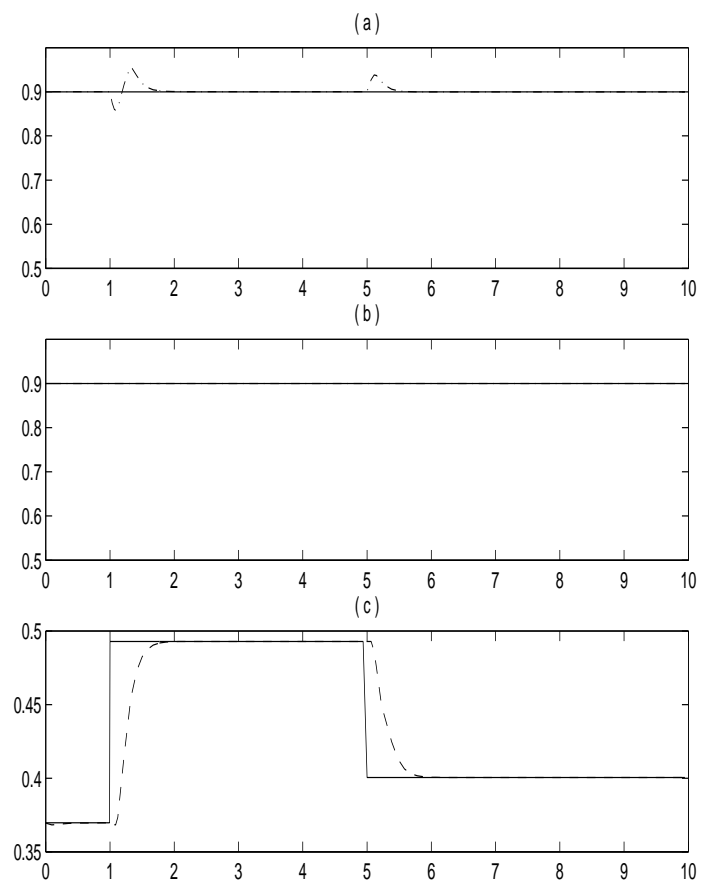

$(2)$

Figure 4. 1a) $V_{t}$ 1b)Control signal 2a) $\left.\left.\hat{\theta}(-),. P_{m}(-) 2 \mathrm{~b}\right) \widehat{P}_{m}(--), P_{m}(-) 2 \mathrm{c}\right) X_{s}(-)$, Computed $X_{s}(--)$

\section{Conclusion}

In this paper, we have treated the problem of exponentially stabilizing a power generator using available output measurement. The proposed controller may be implemented in practice since only actually measured outputs are used for feedback. Usually, nonlinear controllers found in literature need the mechanical power, the transmission line impedance and the power angle, which make them not implementable. On the other hand, the linear controllers, usually implemented in power plants, do not assure a large stability region, and are not able to stand large perturbations.

To design the proposed controller, we have first developed techniques to compute the unknown parameters such that the equilibrium point may be recovered after a fault or parameters changes. We then design trajectories (one for each state) toward this new point that are tracked by the states, driven by the controller. This is achieved by an adaptive output feedback linearization scheme designed using backstepping techniques, that also assures boundedness of all signals. The convergence of the trajectories to the equilibrium point is simultaneous to the convergence of the states toward the trajectories and the generation of these trajectories is made on-line by an exponentially stable adaptive estimator that recovers the mechanical power value.

Finally we present simulation results that corroborate our claims. They show the good behavior of all states, outputs and control signal even in the presence of severe faults on 
turbine and on transmission line.

As further developments, our main goal is to extend these results to the multi-machine case. Actually, the single-machine study is a step toward the more general (and in practice the most important) case of multiple interconnected generators undergoing interzone oscillations. Since, in general, power plants are located very far from each others, centralized controllers that need information from each machine in the system are not realistic. The scheme proposed in this paper could be a starting point in the design of decentralized controllers.

\section{REFERENCES}

1. A. Bazanella, A. S. Silva, and P. Kokotovic. Lyapunov design of excitation control for synchronous machines. In Proc. 36th IEEE - CDC, San Diego, CA, 1997. IEEE.

2. A. R. Bergen. Power Systems Analysis. Prentice Hall, Englewood Cliffs, NJ, 2000.

3. H. Bourles, S. Peres, T. Margotin, and M.P. Hourry. Analysis and design of a robust coordinated avr/pss. IEEE Trans. on Power Systems, 13, No. 2:568-575, 1998.

4. G.R. Damm. Contributions to the Stabilization of Power Generators. PhD thesis, L2S-CNRS-SUPELEC, 2001.

5. G.R. Damm, R. Marino, and F. Lamnabhi-Lagarrigue. Adaptive nonlinear excitation control of synchronous generators. chapter in book - Nonlinear and Adaptive Control, Springer-Verlang, 2002.

6. L. Gao, L. Chen, Y. Fan, and H. Ma. A nonlinear control design for power systems. Automatica, 28:975979,1992

7. E. Irving. Robust adaptive control and applications to electrical generator terminal voltage model reference control. In Procedings of the first Nonlinear Control Network (NCN) Pedagogical School, pages 109-148, Athens, Greece, 6-10, September 1999. NCN.

8. M. Keleman, F. Okou, O. Akhrif, and L.-A. Dessaint. Robust control of a synchronous power generator. chapter in book - Nonlinear Control in the Year 2000, 1:583, 2000.

9. H. Khalil. Nonlinear Systems. Prentice Hall, New Jersey, 1996.

10. P. Kundur. Power System Stability and Control. McGraw-Hill, New York, 1994.

11. R. Marino. An example of nonlinear regulator. IEEE Trans. Automatic Control, 29:276-279, 1984.

12. R. Marino, G.R. Damm, and F. Lamnabhi-Lagarrigue. Adaptive nonlinear excitation control of synchronous generators with unknown mechanical power. chapter in book - Nonlinear Control in the Year 2000, 2000 .

13. R. Marino and P. Tomei. Nonlinear Control Design - Geometric, Adaptive and Robust. Prentice Hall, Hemel Hempstead, 1995.

14. R. Marino and P. Tomei. Robust adaptive state-feedback tracking for nonlinear systems. IEEE Trans. Automatic Control, 43, no. 1:84-89, Jan. 1998.

15. K. S. Narendra and A. M. Annaswamy. Adaptive Systems. Prentice Hall, Englewood Cliffs, NJ, 1989.

16. J. Pomet and L. Praly. Adaptive nonlinear regulation: estimation from the lyapunov equation. IEEE Trans. Automatic Control, 37:729-740, 1992.

17. S. Sastry and M. Bodson. Adaptive Control - Stability, Convergence and Robustness. Prentice Hall, New Jersey, 1989

18. P.W. Sauer, S. Ahmed-Zaid, and P.V. Kokotovic. An integral manifold approach to reduced order dynamic modeling of synchronous machines. IEEE Trans. on Power Systems, 3, No. 1:17-23, February, 1988.

19. Y. Wang and D. J. Hill. Robust nonlinear coordinated control of power systems. Automatica, 32:611-618, 1996.

20. Y. Wang, D. J. Hill, R. H. Middleton, and L. Gao. Transient stability enhancement and voltage regulation of power systems. IEEE Trans. Power Systems, 8:620-627, 1993.

21. Y. Wang, D. J. Hill, R. H. Middleton, and L. Gao. Transient stabilization of power systems with an adaptive control law. Automatica, 30:1409-1413, 1994.

Prepared using rncauth.cls

Int. J. Robust Nonlinear Control 20; :- 


\section{APPENDIX}

In the following, we will compute the terms $\frac{d \delta_{r}}{d \widehat{P}_{m}}, \frac{d^{2} \delta_{r}}{d \widehat{P}_{m}^{2}}, \frac{d^{3} \delta_{r}}{d \widehat{P}_{m}^{3}}, \frac{d \widehat{P}_{m}}{d t}, \frac{d^{2} \widehat{P}_{m}}{d t^{2}}$ and $\frac{d^{3} \widehat{P}_{m}}{d t^{3}}$ in order to build equation (16).

For the sake of simplicity, we first define:

$$
\begin{aligned}
a & =\frac{V_{s}}{X_{s}} \\
b & =\frac{V_{s} X_{d}}{X_{d s}}
\end{aligned}
$$

such that we may rewrite (13) as:

$$
\delta_{r}=\operatorname{arccot}\left(\frac{a\left(-b+\sqrt{V_{t r}^{2}-\frac{\widehat{P}_{m}^{2}}{a^{2}}}\right)}{\widehat{P}_{m}}\right)
$$

Using:

$$
\frac{d \operatorname{arccot}(x)}{d x}=-\frac{1}{1+x^{2}}
$$

we compute

$$
\begin{aligned}
& \frac{d \delta_{r}}{d \widehat{P}_{m}}=-\frac{-\frac{a\left(-b+\sqrt{V_{t r}^{2}-\frac{\hat{P}_{m}^{2}}{a^{2}}}\right)}{\widehat{P}_{m}^{2}}-\frac{1}{a \sqrt{V_{t r}^{2}-\frac{\hat{P}_{m}^{2}}{a^{2}}}}}{1+\frac{a^{2}\left(-b+\sqrt{V_{t r}^{2}-\frac{\hat{P}_{m}^{2}}{a^{2}}}\right)^{2}}{\widehat{P}_{m}^{2}}} \\
& \triangleq\left(N_{1}+N_{2}\right) * \text { Den }
\end{aligned}
$$

where:

$$
\begin{aligned}
\text { Den } & =-\frac{1}{1+\frac{a^{2}\left(-b+\sqrt{V_{t r}^{2}-\frac{\hat{P}_{m}^{2}}{a^{2}}}\right)^{2}}{\widehat{P}_{m}^{2}}} \\
N_{1} & =-\frac{a\left(-b+\sqrt{V_{t r}^{2}-\frac{\widehat{P}_{m}^{2}}{a^{2}}}\right)}{\widehat{P}_{m}^{2}} \\
N_{2} & =-\frac{1}{a \sqrt{V_{t r}^{2}-\frac{\widehat{P}_{m}^{2}}{a^{2}}}}
\end{aligned}
$$

Now, recalling that: 


$$
\frac{d^{2} \delta_{r}}{d \widehat{P}_{m}^{2}}=\left(\frac{d D e n}{d \widehat{P}_{m}} *\left(N_{1}+N_{2}\right)+\operatorname{Den} *\left(\frac{d N_{1}}{d \widehat{P}_{m}}+\frac{d N_{2}}{d \widehat{P}_{m}}\right)\right)
$$

we first compute:

$$
\begin{aligned}
\frac{d N_{1}}{d \widehat{P}_{m}} & =2 \frac{a\left(-b+\sqrt{V_{t r}^{2}-\frac{\widehat{P}_{m}^{2}}{a^{2}}}\right)}{\widehat{P}_{m}^{3}}+\frac{1}{a \widehat{P}_{m} \sqrt{V_{t r}^{2}-\frac{\widehat{P}_{m}^{2}}{a^{2}}}} \\
\frac{d N_{2}}{d \widehat{P}_{m}} & =-\frac{\widehat{P}_{m}}{a^{3}\left(V_{t r}^{2}-\frac{\widehat{P}_{m}^{2}}{a^{2}}\right)^{\left(\frac{3}{2}\right)}} \\
\frac{d D e n}{d \widehat{P}_{m}} & =\frac{-2 \frac{a^{2}\left(-b+\sqrt{V_{t r}^{2}-\frac{\hat{P}_{m}^{2}}{a^{2}}}\right)^{2}}{\widehat{P}_{m}^{3}}-\frac{2\left(-b+\sqrt{V_{t r}^{2}-\frac{\hat{P}_{m}^{2}}{a^{2}}}\right)}{\left(1+\frac{a^{2}\left(-b+\sqrt{V_{t r}^{2}-\frac{\widehat{P}_{m}^{2}}{a^{2}}}\right)^{2}}{\widehat{P}_{t r}^{2}-\frac{\widehat{P}_{m}^{2}}{a^{2}}}\right.}}{\widehat{P}_{m}^{2}}
\end{aligned}
$$

and then:

$$
\begin{aligned}
\frac{d^{2} \delta_{r}}{d \widehat{P}_{m}^{2}}= & \frac{\left(-\frac{a\left(-b+\sqrt{V_{t r}^{2}-\frac{\hat{P}_{m}^{2}}{a^{2}}}\right)}{\widehat{P}_{m}^{2}}-\frac{1}{a \sqrt{V_{t r}^{2}-\frac{\hat{P}_{m}^{2}}{a^{2}}}}\right)\left(-2 \frac{a^{2}\left(-b+\sqrt{V_{t r}^{2}-\frac{\hat{P}_{m}^{2}}{a^{2}}}\right)^{2}}{\widehat{P}_{m}^{3}}-\frac{2\left(-b+\sqrt{V_{t r}^{2}-\frac{\hat{P}_{m}^{2}}{a^{2}}}\right)}{\widehat{P}_{m} \sqrt{V_{t r}^{2}-\frac{\hat{P}_{m m}^{2}}{a^{2}}}}\right)}{\left(1+\frac{a^{2}\left(-b+\sqrt{V_{t r}^{2}-\frac{\hat{P}_{m}^{2}}{a^{2}}}\right)^{2}}{\widehat{P}_{m}^{2}}\right)^{2}} \\
& -\frac{2 \frac{a\left(-b+\sqrt{V_{t r}^{2}-\frac{\hat{P}_{m}^{2}}{a^{2}}}\right)}{\widehat{P}_{m}^{3}}+\frac{1}{a \widehat{P}_{m} \sqrt{V_{t r}^{2}-\frac{\hat{P}_{m}^{2}}{a^{2}}}}-\frac{\widehat{P}_{m}}{a^{3}\left(V_{t r}^{2}-\frac{\hat{P}_{m}^{2}}{a^{2}}\right)^{\left(\frac{3}{2}\right)}}}{1+\frac{a^{2}\left(-b+\sqrt{V_{t r}^{2}-\frac{\hat{P}_{m}^{2}}{a^{2}}}\right)^{2}}{\widehat{P}_{m}^{2}}} \\
& \triangleq M_{1} * M_{2} * M_{3}+M_{4} * M_{5}
\end{aligned}
$$

Here again we have split this equation such that $M_{i}, i=1 . .5$ as well as its derivatives are defined as:

Prepared using rncauth.cls

Int. J. Robust Nonlinear Control 20; :- 


$$
\begin{aligned}
& M 1=\frac{1}{\left(1+\frac{a^{2}\left(-b+\sqrt{V_{t r}^{2}-\frac{\hat{P}_{m}^{2}}{a^{2}}}\right)^{2}}{\widehat{P}_{m}^{2}}\right)^{2}} \\
& M 2=-2 \frac{a^{2}\left(-b+\sqrt{V_{t r}^{2}-\frac{\hat{P}_{m}^{2}}{a^{2}}}\right)^{2}}{\widehat{P}_{m}^{3}}-\frac{2\left(-b+\sqrt{V_{t r}^{2}-\frac{\hat{P}_{m}^{2}}{a^{2}}}\right)}{\widehat{P}_{m} \sqrt{V_{t r}^{2}-\frac{\widehat{P}_{m}^{2}}{a^{2}}}} \\
& M 3=-\frac{a\left(-b+\sqrt{V_{t}^{2}-\frac{\widehat{P}_{m}^{2}}{a^{2}}}\right)}{\widehat{P}_{m}^{2}}-\frac{1}{a \sqrt{V_{t}^{2}-\frac{\widehat{P}_{m}^{2}}{a^{2}}}} \\
& M 4=-\frac{1}{1+\frac{a^{2}\left(-b+\sqrt{V_{t r}^{2}-\frac{\hat{P}_{m}^{2}}{a^{2}}}\right)^{2}}{\hat{P}_{m}^{2}}} \\
& M 5=2 \frac{a\left(-b+\sqrt{V_{t}^{2}-\frac{\widehat{P}_{m}^{2}}{a^{2}}}\right)}{\widehat{P}_{m}^{3}}+\frac{1}{a \widehat{P}_{m} \sqrt{V_{t r}^{2}-\frac{\widehat{P}_{m}^{2}}{a^{2}}}}-\frac{\widehat{P}_{m}}{a^{3}\left(V_{t}^{2}-\frac{\widehat{P}_{m}^{2}}{a^{2}}\right)^{\left(\frac{3}{2}\right)}}
\end{aligned}
$$

and:

$$
\begin{aligned}
& \frac{d M_{1}}{d \widehat{P}_{m}}=-2 \frac{-2 \frac{a^{2}\left(-b+\sqrt{V_{t r}^{2}-\frac{\hat{P}_{m}^{2}}{a^{2}}}\right)^{2}}{\widehat{P}_{m}^{3}}-\frac{2\left(-b+\sqrt{V_{t r}^{2}-\frac{\hat{P}_{m}^{2}}{a^{2}}}\right)}{\widehat{P}_{m} \sqrt{V_{t r}^{2}-\frac{\hat{P}_{m}^{2}}{a^{2}}}}}{\left(1+\frac{a^{2}\left(-b+\sqrt{V_{t r}^{2}-\frac{\hat{P}_{m}^{2}}{a^{2}}}\right)^{2}}{\widehat{P}_{m}^{2}}\right)^{3}} \\
& \frac{d M_{2}}{d \widehat{P}_{m}}=6 \frac{a^{2}\left(-b+\sqrt{V_{t r}^{2}-\frac{\widehat{P}_{m}^{2}}{a^{2}}}\right)^{2}}{\widehat{P}_{m}^{4}}+\frac{6\left(-b+\sqrt{V_{t r}^{2}-\frac{\widehat{P}_{m}^{2}}{a^{2}}}\right)}{\widehat{P}_{m}^{2} \sqrt{V_{t r}^{2}-\frac{\widehat{P}_{m}^{2}}{a^{2}}}}+\frac{2}{\left(V_{t r}^{2}-\frac{\widehat{P}_{m}^{2}}{a^{2}}\right) a^{2}}-\frac{2\left(-b+\sqrt{V_{t r}^{2}-\frac{\widehat{P}_{m}^{2}}{a^{2}}}\right)}{\left(V_{t}^{2}-\frac{\widehat{P}_{m}^{2}}{a^{2}}\right)^{\left(\frac{3}{2}\right)} a^{2}} \\
& \frac{d M_{3}}{d \widehat{P}_{m}}=2 \frac{a\left(-b+\sqrt{V_{t r}^{2}-\frac{\widehat{P}_{m}^{2}}{a^{2}}}\right)}{\widehat{P}_{m}^{3}}+\frac{1}{a \widehat{P}_{m} \sqrt{V_{t r}^{2}-\frac{\widehat{P}_{m}^{2}}{a^{2}}}}-\frac{\widehat{P}_{m}}{a^{3}\left(V_{t r}^{2}-\frac{\widehat{P}_{m}^{2}}{a^{2}}\right)^{\left(\frac{3}{2}\right)}}
\end{aligned}
$$




$$
\frac{d M_{4}}{d \widehat{P}_{m}}=\frac{\left.-2 \frac{a^{2}\left(-b+\sqrt{V_{t r}^{2}-\frac{\hat{P}_{m}^{2}}{a^{2}}}\right)^{2}}{\widehat{P}_{m}^{3}}-\frac{2\left(-b+\sqrt{V_{t r}^{2}-\frac{\hat{P}_{m}^{2}}{a^{2}}}\right)}{\left(1+\frac{a^{2}\left(-b+\sqrt{V_{t r}^{2}-\frac{\hat{P}_{m}^{2}}{a^{2}}}\right)^{2}}{\widehat{P}_{t r}^{2}-\frac{\hat{P}_{m}^{2}}{a^{2}}}\right.}\right)^{2}}{(1+2)}
$$

$$
\frac{d M_{5}}{d \widehat{P}_{m}}=-6 \frac{a\left(-b+\sqrt{V_{t r}^{2}-\frac{\widehat{P}_{m}^{2}}{a^{2}}}\right)}{\widehat{P}_{m}^{4}}-\frac{3}{a \widehat{P}_{m}^{2} \sqrt{V_{t r}^{2}-\frac{\widehat{P}_{m}^{2}}{a^{2}}}}-\frac{3 \widehat{P}_{m}^{2}}{a^{5}\left(V_{t r}^{2}-\frac{\widehat{P}_{m}^{2}}{a^{2}}\right)^{\left(\frac{5}{2}\right)}}
$$

The third derivative of $\delta_{r}$ with respect to $\widehat{P}_{m}$ is then given by:

$$
\frac{d^{3} \delta_{r}}{d \widehat{P}_{m}^{3}}=\frac{d M_{1}}{d \widehat{P}_{m}} * M_{2} * M_{3}+M_{1} * \frac{d M_{2}}{d \widehat{P}_{m}} * M_{3}+M 1 * M_{2} * \frac{d M_{3}}{d \widehat{P}_{m}}+\frac{d M_{4}}{d \widehat{P}_{m}} * M_{5}+M_{4} * \frac{d M_{5}}{d \widehat{P}_{m}}
$$

Its complete expression being: 


$$
\begin{aligned}
& \frac{d^{3} \delta_{r}}{d \widehat{P}_{m}^{3}}=-\frac{-6 \frac{a\left(-b+\sqrt{V_{t r}^{2}-\frac{\hat{P}_{m}^{2}}{a^{2}}}\right)}{\widehat{P}_{m}^{4}}-\frac{3}{a \widehat{P}_{m}^{2} \sqrt{V_{t r}^{2}-\frac{\hat{P}_{m n}^{2}}{a^{2}}}}-\frac{3 \widehat{P}_{m}^{2}}{a^{5}\left(V_{t r}^{2}-\frac{\widehat{P}_{m}^{2}}{a^{2}}\right)^{\left(\frac{5}{2}\right)}}}{1+\frac{a^{2}\left(-b+\sqrt{V_{t r}^{2}-\frac{\hat{P}_{m}^{2}}{a^{2}}}\right)^{2}}{\widehat{P}_{m}^{2}}} \\
& +\frac{2}{\left(1+\frac{a^{2}\left(-b+\sqrt{V_{t r}^{2}-\frac{\hat{P}_{m}^{2}}{a^{2}}}\right)^{2}}{\widehat{P}_{m}^{2}}\right)^{2}} *\left(-2 \frac{a^{2}\left(-b+\sqrt{V_{t r}^{2}-\frac{\widehat{P}_{m}^{2}}{a^{2}}}\right)^{2}}{\widehat{P}_{m}^{3}}-\frac{2\left(-b+\sqrt{V_{t r}^{2}-\frac{\widehat{P}_{m}^{2}}{a^{2}}}\right)}{\widehat{P}_{m} \sqrt{V_{t r}^{2}-\frac{\widehat{P}_{m}^{2}}{a^{2}}}}\right) \\
& *\left(2 \frac{a\left(-b+\sqrt{V_{t r}^{2}-\frac{\widehat{P}_{m}^{2}}{a^{2}}}\right)}{\widehat{P}_{m}^{3}}+\frac{1}{a \widehat{P}_{m} \sqrt{V_{t r}^{2}-\frac{\widehat{P}_{m}^{2}}{a^{2}}}}-\frac{\widehat{P}_{m}}{a^{3}\left(V_{t r}^{2}-\frac{\widehat{P}_{m}^{2}}{a^{2}}\right)^{\left(\frac{3}{2}\right)}}\right)
\end{aligned}
$$

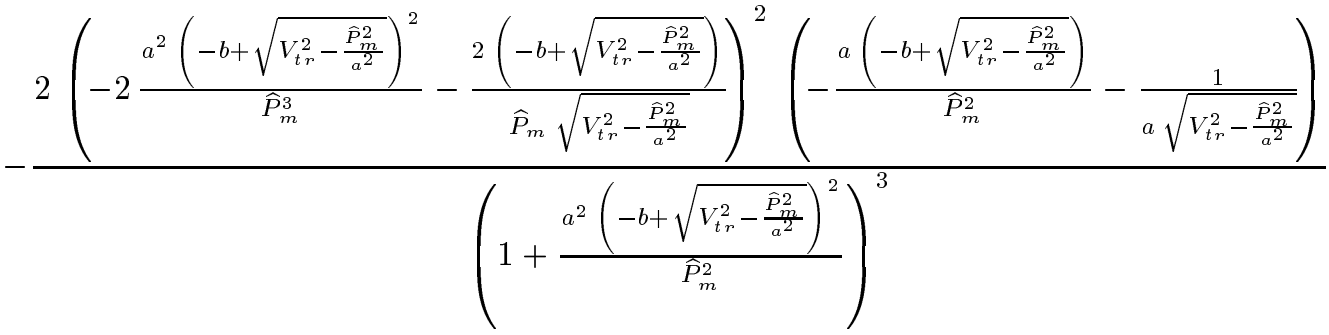

$$
\begin{aligned}
& +\frac{1}{\left(1+\frac{a^{2}\left(-b+\sqrt{V_{t r}^{2}-\frac{\hat{P}_{m}^{2}}{a^{2}}}\right)^{2}}{\widehat{P}_{m}^{2}}\right)^{2}}\left[\left(6 \frac{a^{2}\left(-b+\sqrt{V_{t r}^{2}-\frac{\widehat{P}_{m}^{2}}{a^{2}}}\right)^{2}}{\widehat{P}_{m}^{4}}+\frac{6\left(-b+\sqrt{V_{t r}^{2}-\frac{\widehat{P}_{m}^{2}}{a^{2}}}\right)}{\widehat{P}_{m}^{2} \sqrt{V_{t r}^{2}-\frac{\widehat{P}_{m}^{2}}{a^{2}}}}\right.\right. \\
& \left.\left.+\frac{2}{\left(V_{t r}^{2}-\frac{\widehat{P}_{m}^{2}}{a^{2}}\right) a^{2}}-\frac{2\left(-b+\sqrt{V_{t r}^{2}-\frac{\hat{P}_{m}^{2}}{a^{2}}}\right)}{\left(V_{t r}^{2}-\frac{\widehat{P}_{m}^{2}}{a^{2}}\right)^{\left(\frac{3}{2}\right)} a^{2}}\right)\left(-\frac{a\left(-b+\sqrt{V_{t r}^{2}-\frac{\widehat{P}_{m}^{2}}{a^{2}}}\right)}{\widehat{P}_{m}^{2}}-\frac{1}{a \sqrt{V_{t r}^{2}-\frac{\hat{P}_{m}^{2}}{a^{2}}}}\right)\right]
\end{aligned}
$$

Now one may remark in (16) that we need the second and third derivatives of $\widehat{P}_{m}$. These derivatives are not available, as they would imply the exact knowledge of $P_{m}$. To avoid this problem we first remember:

$$
\frac{d \widehat{P}_{m}}{d t}=\gamma_{1} \widetilde{\omega}_{e}
$$

and remarking that:

\section{Prepared using rncauth.cls}




$$
P_{m}=\theta=\widetilde{\theta}+\widehat{\theta}
$$

one may compute:

$$
\begin{aligned}
\frac{d^{2} \widehat{P}_{m}}{d t^{2}} & =\gamma_{1} \dot{\tilde{\omega}}_{e}=\gamma_{1} \frac{\omega_{s}}{H} \widetilde{P}_{m}-\gamma_{1} \frac{D}{H} \widetilde{\omega}_{e} \\
& =\gamma_{1} \frac{\omega_{s}}{H}\left(P_{m}-\widehat{P}_{m}\right)-\gamma_{1} \frac{D}{H} \widetilde{\omega}_{e} \\
& =\gamma_{1} \frac{\omega_{s}}{H}\left(\widehat{\theta}-\widehat{P}_{m}\right)-\gamma_{1} \frac{D}{H} \widetilde{\omega}_{e}+\gamma_{1} \frac{\omega_{s}}{H} \widetilde{\theta}
\end{aligned}
$$

and:

$$
\begin{aligned}
\frac{d^{3} \widehat{P}_{m}}{d t^{3}} & =\gamma_{1} \frac{\omega_{s}}{H} \dot{\widetilde{P}}_{m}-\gamma_{1} \frac{D}{H} \dot{\tilde{\omega}}_{e} \\
& =\gamma_{1} \frac{\omega_{s}}{H}\left(-\gamma_{1} \widetilde{\omega}_{e}\right)-\gamma_{1} \frac{D}{H}\left(\frac{\omega_{s}}{H} \widetilde{P}_{m}-\frac{D}{H} \widetilde{\omega}_{e}\right) \\
& =\left(\gamma_{1} \frac{D^{2}}{H^{2}}-\gamma_{1}^{2} \frac{\omega_{s}}{H}\right) \widetilde{\omega}_{e}-\gamma_{1} \frac{D \omega_{s}}{H^{2}}\left(\widehat{\theta}-\widehat{P}_{m}\right)-\gamma_{1} \frac{D \omega_{s}}{H^{2}} \widetilde{\theta}
\end{aligned}
$$

